hep-th/9705044, IASSNS-HEP-97/40

\title{
New Phases of String Theory and 6d RG Fixed Points via Branes at Orbifold Singularities
}

\author{
Julie D. Blum and Kenneth Intriligator* \\ School of Natural Sciences \\ Institute for Advanced Study \\ Princeton, NJ 08540, USA
}

\begin{abstract}
We discuss type II and type I branes at general $A D E$ type orbifold singularities. We show that there are new phases of type I or heterotic string theory in six dimensions, involving extra tensor multiplets, which arise when small instantons sit on orbifold singularities. The theories with extra tensor multiplets are explicitly constructed via orientifolds. The world-volume theories in type IIB or type I five-branes at orbifold singularities lead to the existence of several infinite classes of six dimensional, interacting, renormalization group fixed point theories.
\end{abstract}

$5 / 97$

* On leave 1996-1997 from Department of Physics, University of California, San Diego. 


\section{Introduction}

The small $E_{8}$ instanton is qualitatively different from the small $S O(32)$ instanton. In both cases, the small instanton is at a point $p \in \mathbb{R}^{4}$ and thus yields a $5+1$ dimensional world volume theory with minimal $\mathcal{N}=(1,0)$ supersymmetry ( 8 supercharges). In the $S O(32)$ case, the 6d world-volume theory is a $S p(1)$ gauge theory [1], which is IR free. On the other hand, the $E_{8}$ case is more interesting: at the point in the moduli space where the $E_{8}$ instanton is of zero size, there is a transition to another "Coulomb branch" moduli space of the theory, labeled by the expectation value $\langle\Phi\rangle \in \mathbb{R}^{+}$of a real scalar in a tensor multiplet. The branch with the tensor multiplet corresponds in $M$ theory to a phase in which the zero size instanton has become a five-brane which has moved a distance $\langle\Phi\rangle$ into the 11th dimension [2, 3:4]. At the transition point between the two branches, where $\langle\Phi\rangle \rightarrow 0$, there appears to be a "tensionless string" [5, 3, 4, 6] - though there is evidence that it is an interacting local quantum field theory at a non-trivial RG fixed point. The existence of other non-trivial fixed points of the renormalization group in six dimensions was discussed in [7,8,9, [10].

A situation similar to that of the small $E_{8}$ instanton occurs when the small $S O(32)$ instanton sits, not at a smooth point on the transverse $\mathbb{R}^{4}$ but, rather, at a $\mathbb{Z}_{M}$ orbifold singularity. There are new phases with extra tensor multiplets and the world-volume theory leads to non-trivial $6 \mathrm{~d}$ super-conformal field theories $[\mathbb{8}]$. For a $\mathbb{Z}_{2}$ orbifold singularity, the new phase has a single extra tensor multiplet and was found via $F$ theory in [11]. The new phases for $\mathbb{Z}_{M}$ singularities were given an orientifold construction in [12].

Here we consider what happens when small $S O(N)$ instantons sit on general orbifold singularities. The result will be many more new phases for the $S O(32)$ heterotic or type I string theories. In addition, the world-volume theories will lead to the existence of an infinite number of new classes of non-trivial $6 \mathrm{~d}$ superconformal field theories. We also point out that type IIB five-branes at orbifold singularities lead to an infinite number of classes of non-trivial $6 \mathrm{~d}$ superconformal field theories.

In the next section we discuss some general preliminaries. In the first subsection, we review ALE spaces and their hyper-Kahler quotient construction [13]. As discussed in [14, [15], these theories have a physical realization via branes on the corresponding ALE space. In the next subsection we discuss general aspects of instantons on ALE spaces. In sect. 3 we briefly review the situation for $U(N)$ instantons on ALE spaces and the hyper-Kahler quotient construction of their moduli spaces [16]. It is worth emphasizing 
that the construction of [16] applies only for gauge group $U(N)$. In sect. 3.2 we mention the orbifold construction of these theories and how tadpole conditions [12] yield a relation of [16]. Using a relation [12] between first Chern classes and the NSNS $B$ field, this allows us to determine the value of the $B$ field in the orbifold construction.

In sect. 4 we discuss $K$ IIB five-branes at a $\mathbb{C}^{2} / \Gamma_{G}$ orbifold singularity. The $6 \mathrm{~d}$ world-volume theory is a $\mathcal{N}=(1,0)$ supersymmetric theory with gauge group and matter content given by a "moose1]" (or "quiver") diagram, which is the extended Dynkin diagram of the $A D E$ group $G$ corresponding to the $\Gamma_{G} \subset S U(2)$, along with $r=\operatorname{rank}(G)$ extra tensor multiplets. The case $\Gamma_{G}=\mathbb{Z}_{2}$ gives a theory mentioned in [18] as a solution of the anomaly cancellation conditions. We argue that these theories yield six-dimensional, interacting, scale invariant theories for every discrete $\Gamma_{G} \subset S U(2)$, and for any $K$.

In sect. 5 we discuss $S O(N)$ instantons on $\mathbb{C}^{2} / \Gamma_{G}$ ALE spaces. We present supersymmetric gauge theories whose Higgs branches we argue give hyper-Kahler quotient constructions of the moduli space of $S O(N)$ instantons on general ALE spaces, albeit with a caveat about some of the ALE blowing up modes being frozen to zero. The theories are based on particular products of classical gauge groups arranged according to moose diagrams which are again related to the extended Dynkin diagrams of the $A D E$ gauge group $G$. This construction applies for any $S O(N)$. These particular theories, however, could not arise physically in the world-volume of D5 branes at the orbifold singularity, as they suffer from deadly $6 \mathrm{~d}$ gauge anomalies. For the special case of $S O(32)$ there is a modified version of the gauge theories, with altered relations between the gauge groups and matter content and also with additional tensor multiplets, which we argue does arise physically in the world-volume of the D5 branes. These world-volume theories arise in a "Coulomb branch" of the full moduli space, in which $29 n_{T}$ hypermultiplets are traded for $n_{T}$ tensor multiplets, much as in the small $E_{8}$ instanton. The origin of the Coulomb branch yields, for every ALE space, an infinite family of non-trivial, six dimensional, interacting, scale invariant theories.

In sect. 6 we give an orientifold construction of these theories, showing directly the existence of the new phases with extra tensor multiplets.

In sect. 7 we discuss other $6 \mathrm{~d}$ gauge theories, which are similar to those discussed in sect. 5, but without coupling between the five-branes and the nine-branes. The fivebranes thus do not have an interpretation as small instantons, and strings ending on these

1 in the terminology of [17]. 
branes do not have Dirichlet boundary conditions but rather twisted boundary conditions. Including these branes may resolve the puzzle of anomaly cancellation in the theories discussed by [19]. One of these theories, with gauge group given by the $A_{1}$ extended Dynkin diagram moose, was previously found by [18]. In sect. 7.1 we discuss the orientifold construction of this theory.

Some general observations about anomaly restrictions on $6 \mathrm{~d}$ gauge theories based on product groups are made in sect. 8. Finally, in an appendix we include a few relevant facts about the representations of the discrete subgroups of $S U(2)$.

Note added: Related results concerning small $\operatorname{Spin}(32)$ instantons at $D$ and $E$ type singularities have been independently obtained via $F$ theory in [20]. We thank the authors of [20] for comparing their results with ours, thereby finding a typo in the original version of this paper concerning the representations of $\Gamma_{E_{7}}$.

\section{General preliminaries}

\subsection{ALE spaces and branes}

The ALE spaces are solutions of Einstein's equations described as possibly blown up orbifolds $\mathbb{C}^{2} / \Gamma_{G}$, with $\Gamma_{G} \subset S U(2)$ acting on the $S^{3}$ at infinity. The discrete $S U(2)$ subgroups: cyclic, dihedral, tetrahedral, octahedral, and icosahedral, have a famous correspondence with, respectively, the $A_{r}, D_{r}, E_{6}, E_{7}$, and $E_{8}$ groups. For example, the nodes $\mu=0 \ldots r=\operatorname{rank}(G)$ of the extended Dynkin diagram of the $A D E$ group $G$ correspond to the irreducible representations $R_{\mu}$ of $\Gamma_{G}$, with $\left|R_{\mu}\right|=n_{\mu}$, the Dynkin indices; therefore $\left|\Gamma_{G}\right|=n_{\mu} n_{\mu}$ (throughout greek indices run from $0 \ldots r$ and repeated indices are summed). Also, $R_{Q} \times R_{\mu}=a_{\mu \nu} R_{\nu}$ where $R_{Q}$ is the fundamental two dimensional representation given by $\Gamma_{G} \subset S U(2)$ and $a_{\mu \nu}$ is one if nodes $\mu$ and $\nu$ are linked in the extended Dynkin diagram and zero otherwise. The character of this relation is

$$
\chi_{Q}(g) \chi_{\mu}(g)=a_{\mu \nu} \chi_{\nu}(g),
$$

for all $g \in \Gamma_{G}$. In particular, for $g=1$, this gives $\widetilde{C}_{\mu \nu} n_{\nu}=0$, where $\widetilde{C}_{\mu \nu}=2 \delta_{\mu \nu}-a_{\mu \nu}$ is the extended Cartan matrix; the Dynkin indices $n_{\mu}$ give the only null vector of $\widetilde{C}_{\mu \nu}$.

The ALE space $X \cong \mathbb{C}^{2} / \Gamma_{G}$ has $r$ non-trivial two cycles $\Sigma_{i}, i=1 \ldots r$, generating $H_{2} . X$ is hyper-Kahler with a triple of Kahler forms $\vec{\omega}$ and

$$
\int_{\Sigma_{i}} \vec{\omega}=\vec{\zeta}_{i}
$$


with $\vec{\zeta}_{i}, i=1 \ldots r$, the $3 r$ blowing up parameters.

The ALE spaces have a hyper-Kahler quotient construction [13]; i.e. there are supersymmetric gauge theories (with 8 super-charges) which have the ALE spaces as their

Higgs branches. The theories have gauge group $\prod_{\mu=0}^{r} U\left(n_{\mu}\right) / U(1)$, where the $U(1)$ in the quotient is the overall $U(1)$. The matter content of the gauge theory is given by hypermultiplets in the representations $\frac{1}{2} \oplus_{\mu \nu=0}^{r} a_{\mu \nu}\left(\square_{\mu}, \bar{\square}_{\nu}\right)$, where the index labels the gauge group and the $\frac{1}{2}$ is to avoid a double counting. Throughout, $n_{\mu}$ and $a_{\mu \nu}$ are defined as above. The $3 r$ blowing parameters $\vec{\zeta}_{i}$ enter as the $r$ Fayet-Iliopoulos parameters which can be included for the $r U(1)$ factors in the gauge group [13.

The above gauge theories arise physically, for example in $D 0$ branes (of IIA) or D1 branes (of IIB), which probe the geometry of the ALE spaces [14,15].

\subsection{Instantons on ALE spaces}

On flat $\mathbb{R}^{4}$, instantons in an arbitrary gauge group $\mathcal{H}$ (not to be confused with the ADE group $G$ in $\left.\mathbb{C}^{2} / \Gamma_{G}\right)$ are topologically specified by the instanton number $K$, which is a nonnegative integer associated with $\pi_{3}(\mathcal{H}) \cong \mathbb{Z}$. The moduli space $\mathcal{M}_{\text {Inst }}$ of instantons with a given $K$ is a hyper-Kahler space with dimension in hypermultiplet (i.e quaternionic) units (one quarter of the real dimension) given, using the index theorem of [22], by $\operatorname{dim}\left(\mathcal{M}_{\text {Inst }}\right)=$ $C_{2}(\mathcal{H}) K$, where $\mathcal{C}_{2}(\mathcal{H})$ is the dual Coxeter number (index of the adjoint; e.g. $C_{2}=N$ for $S U(N)$ or $N-2$ for $S O(N))$.

When the usual $\mathbb{R}^{4}$ is replaced with an ALE space $X \cong \mathbb{C}^{2} / \Gamma_{G}$, instantons are specified by certain topological data in addition to the integer $K$. An instanton gauge connection is asymptotically flat and thus usually trivial since the asymptotic space $X_{\infty}$ surrounding the instanton usually has trivial $\pi_{1}$. However, on the ALE space, $\pi_{1}\left(X_{\infty}\right)=\Gamma_{G}$ and thus there can be non-trivial Wilson lines at infinity, leading to non-trivial group elements $\rho_{\infty} \in \mathcal{H}$, representing $\Gamma_{G}$ in the gauge group. Thus, in addition to $K$, the instanton is topologically characterized by integers $w_{\mu}$ in $\rho_{\infty}=\oplus_{\mu} w_{\mu} R_{\mu}$, giving the representation of $\Gamma_{G}$ in $\mathcal{H}$ in terms of the irreps $R_{\mu}$. When the gauge group $\mathcal{H}$ has an Abelian factor, additional topological data is needed to specify the instanton: because $X$ has non-trivial two cycles $\Sigma_{i}, i=1 \ldots r=\operatorname{rank}(G)$, there can be non-trivial first Chern classes $u_{i}=\int_{\Sigma_{i}}(\operatorname{Tr} F / 2 \pi)$. For simplicity, and because we are chiefly in the case of $\mathcal{H}=S O(N)$ where $\operatorname{Tr} F=0$, we will set the $u_{i}=0$ in this section. 
The instanton number (second Chern class) with physical data $K$ and $\rho_{\infty}$ is

$$
I=K+\frac{1}{\kappa\left|\Gamma_{G}\right|} G_{\mu \nu} n_{\mu} w_{\nu}
$$

where $\kappa$ is the index of the embedding of $S U(2)$ in the gauge group $\mathcal{H}$ (e.g. $\kappa=1$ for $S U(N)$ and $\kappa=2$ for $S O(N))$ and $G_{\mu \nu}$ is defined by

$$
G_{0 \nu} \equiv G_{\mu 0} \equiv 0, \quad G_{i j} \equiv C_{i j}^{-1} \quad i, j \neq 0
$$

with $C^{-1}$ the inverse Cartan matrix of the $A D E$ gauge group $G$. Explicit expressions for the $G_{\mu \nu}$ for all gauge groups can be found, for example, in table 7 of [23]. $G_{\mu \nu}$ inverts the extended Cartan matrix $\widetilde{C}_{\mu \nu}$ up to its null vector $n_{\mu}$ :

$$
\widetilde{C}_{\mu \nu} A_{\nu}=B_{\mu} \quad \rightarrow \quad n \cdot B=0 \quad \text { and } \quad A_{\mu}=\alpha n_{\mu}+G_{\mu \nu} B_{\nu}
$$

where $\alpha$ is arbitrary.

The moduli space of instantons on the ALE space is again hyper-Kahler, with dimension which can be determined by the index theorem of [24 for manifolds with boundary (in this case at infinity). In hyper-multiplet units, the dimension is given by

$$
\operatorname{dim}\left(\mathcal{M}_{\text {Inst }}\right)=C_{2}(\mathcal{H}) I+\frac{1}{2\left|\Gamma_{G}\right|} \sum_{g \neq 1} \frac{\chi_{A d}\left(\rho_{\infty}\right)(g)-|\mathcal{H}|}{2-\chi_{Q}(g)}
$$

where the last term is the eta invariant, with the sum over $g \in \Gamma_{G}$, omitting the identity $g=1$. In computing the eta invariant, it will be useful to define

$$
X_{\mu \nu} \equiv \frac{1}{2\left|\Gamma_{G}\right|} \sum_{g \neq 1} \frac{\chi_{\mu}(g) \overline{\chi_{\nu}(g)}-n_{\mu} n_{\nu}}{2-\chi_{Q}(g)}=\frac{1}{2} G_{\mu \nu}-\frac{1}{2\left|\Gamma_{G}\right|}\left(G_{\mu \sigma} n_{\sigma} n_{\nu}+G_{\nu \sigma} n_{\sigma} n_{\mu}\right)
$$

The identity in (2.7) follows from (2.5) and

$$
\widetilde{C}_{\mu \sigma} X_{\sigma \nu}=\frac{1}{2\left|\Gamma_{G}\right|}\left(\left|\Gamma_{G}\right| \delta_{\mu \nu}-n_{\mu} n_{\nu}\right)
$$

which follows easily from (2.1) and character orthogonality. 


\section{Type II: $U(N)$ instantons on ALE spaces.}

\subsection{General aspects}

$N$ coincident type II $p+4$ branes have a supersymmetric $U(N)$ gauge theory living in their world-volume 25]. $K$ coincident type II $p$ branes living inside of this system have a supersymmetric (with eight super-charges) $U(K)$ gauge theory living in their world volume with $N$ matter hypermultiplets in the $\mathbf{K}$ and one in the adjoint $\mathbf{K}^{\mathbf{2}}$. This configuration of branes has the interpretation as $K U(N)$ instantons and, indeed, this $U(K)$ worldvolume theory has a Higgs branch which gives the hyper-Kahler quotient construction of the moduli space of $K U(N)$ instantons [26].

Consider now the situation where the $p$ branes are on an ALE space $\mathbb{C}^{2} / \Gamma_{G}$. The resulting world-volume theory should have a Higgs branch which is isomorphic to the moduli space of $U(N)$ instantons on the ALE space. This moduli space does indeed have a hyper-Kahler quotient construction [16], i.e. a realization as the Higgs branch of a supersymmetric gauge theory with 8 supercharges. It is natural to expect that these theories arise in the world-volume of the $p$ branes on the ALE space; this was shown for the $\mathbb{C}^{2} / \mathbb{Z}_{r+1}$ orbifolds in 14 .

As discussed in the previous section, on an ALE space an instanton can have a nontrivial $\rho_{\infty} \in U(N)$ representing $\Gamma_{G}: \rho_{\infty}=\oplus_{\mu} w_{\mu} R_{\mu}$, where the integers $w_{\mu}$ must satisfy $w \cdot n \equiv w_{\mu} n_{\mu}=N$ in order to have $\rho_{\infty} \in U(N)$. The group element $\rho_{\infty}$ breaks $U(N) \rightarrow$ $\prod_{\mu=0}^{r} U\left(w_{\mu}\right)$. In addition, there can be non-trivial first Chern classes $u_{i}=\int_{\Sigma_{i}}(\operatorname{Tr} F / 2 \pi)$, $i=1 \ldots r$. As discussed in 12 , the $u_{i}$ are related to the integral of the NSNS $B$ field by

$$
u_{i}=N \int_{\Sigma_{i}} B
$$

The theory of [16 has gauge group $\prod_{\mu=0}^{r} U\left(v_{\mu}\right)$, with matter content consisting of $w_{\mu}$ hypermultiplets in the $\square_{\mu}$ and hypermultiplets in the $\frac{1}{2} \oplus_{\mu \nu} a_{\mu \nu}\left(\square_{\mu}, \overline{\square_{\nu}}\right)$, where the subscripts label the gauge group (and the $\frac{1}{2}$ is to avoid double counting). The $v_{\mu}$ are determined in terms of the physical data by

$$
\widetilde{C}_{\mu \nu} v_{\nu}=w_{\mu}-u_{\mu}
$$

$u_{\mu>0}$ are the first Chern classes and $u_{0}$ is determined by $n \cdot u=n \cdot w$, which follows from (3.2). Of course it is physically necessary that the small instanton gauge group is generically completely broken on the Higgs branch; this is indeed the case given the relation 
(3.2) and that all $u_{\mu} \geq 0$. The dimension of the Higgs branch $\mathcal{M}_{H}$ in hypermultiplet (i.e quaternionic) units (one quarter of the real dimension) is given by

$$
\operatorname{dim}\left(\mathcal{M}_{H}\right)=v_{\mu} w_{\mu}-\frac{1}{2} \widetilde{C}_{\mu \nu} v_{\mu} v_{\nu}=\frac{1}{2} v_{\mu}\left(w_{\mu}+u_{\mu}\right)
$$

Note that there are $r+1 U(1)$ factors in the gauge group. The diagonally embedded $U(1)$ factor has no charged matter and thus decouples. Including Fayet-Iliopoulos terms $\vec{\zeta}_{i}, i=1 \ldots r$, for the remaining $r U(1)$ factors gives the dependence of the moduli space of instantons on the $3 r$ blowing up modes of the ALE space. We emphasize again that the construction of [16] applies only for gauge group $U(N)$.

We now introduce some formulae which we will find analogs for $S O(N)$ instantons; for this purpose it suffices to restrict our attention to $u_{\mu>0}=0$. The instanton number (second Chern class) with physical data $K$ and $\rho_{\infty}$ is given by (2.3) with $\kappa=1$; thus

$$
I=\frac{v_{\mu} n_{\mu}}{\left|\Gamma_{G}\right|}
$$

which, as expected, gives the instanton number as the number of $p$ branes on the orbifold. The dimension of the moduli space $\mathcal{M}_{\text {Inst }}$ of instantons is given by $(2.6)$ with $C_{2}(U(N))=$ $N$ and $\chi_{A d}\left(\rho_{\infty}\right)(g)=w_{\mu} w_{\nu} \chi_{\mu}(g) \overline{\chi_{\nu}(g)}$. Thus

$$
\operatorname{dim}\left(\mathcal{M}_{\text {Inst }}\right)=N I+w_{\mu} w_{\nu} X_{\mu \nu}
$$

with $X_{\mu \nu}$ defined as in (2.7). Using (3.4), (3.2), and (2.8), it is easily verified that $\operatorname{dim}\left(\mathcal{M}_{\text {Inst }}\right)$, given by (3.5), agrees with $\operatorname{dim}\left(\mathcal{M}_{H}\right)$, given by (3.3), giving a check of the equivalence $\mathcal{M}_{H} \cong \mathcal{M}_{\text {Inst }}$ proven in [16] for $U(N)$ instantons.

\subsection{Orbifold constructions}

The above gauge theories can be directly obtained from an orbifold construction of the theory on $\mathbb{C}^{2} / \Gamma_{G}$; for the case of $\Gamma_{G}=\mathbb{Z}_{r+1}$ this was shown in [14]. As discussed in [12], the relation (3.2) arises from a tadpole consistency condition. We take $\Gamma_{G}$ to act on the $p$ brane and $p+4$ brane Chan-Paton factors as

$$
\gamma_{g, p}=\oplus_{\mu} R_{\mu}(g) \otimes I_{v_{\mu}}, \quad \gamma_{g, p+4}=\oplus_{\mu} R_{\mu}(g) \otimes I_{w_{\mu}}
$$

where $I_{n}$ is the $n \times n$ identity matrix. Following the discussion in [12], the relevant tadpole equations are those twisted by $g \neq 1$, which are

$$
\frac{1}{2-\chi_{Q}(g)}\left(w_{\mu} \chi_{\mu}(g)-\left(2-\chi_{Q}(g)\right) v_{\mu} \chi_{\mu}(g)\right)^{2}=0 \text {. }
$$


These can be put in the form (3.2), though with a specific value for the $u_{\mu}: u_{\mu}=N n_{\mu} /\left|\Gamma_{G}\right|$. As discussed in [12], this means that the orbifold construction gives a specific value for the integral of the NSNS $B$ field over the $\Sigma_{i}$

$$
\int_{\Sigma_{i}} B=\frac{n_{i}}{\left|\Gamma_{G}\right|}, \quad i=1 \ldots r
$$

The fact that the $B$ field is restricted to a particular value in the weakly coupled orbifold construction is standard, as in [27]. The particular value (3.8) agrees (only) for the $A_{r}$ cases with the result of [21], where the result was obtained by requiring $D 0$ branes to have mass $1 / C_{2}(G) g_{s}$, where $C_{2}(G)=\sum_{\mu} n_{\mu}$.

\section{Type IIB five-branes near a $\mathbb{C}^{2} / \Gamma_{G}$ singularity}

$K$ type IIB five-branes at a point in $\mathbb{R}^{4}$ have a $6 d \mathcal{N}=(1,1)$ supersymmetric gauge theory, with gauge group $U(K)$, living in their world-volume. This theory is anomaly free with arbitrary gauge coupling $g$ and is IR free. Now consider the situation when the fivebranes sit not at a generic point in $\mathbb{R}^{4}$ but at a $\mathbb{C}^{2} / \Gamma_{G}$ orbifold singularity. Much as in [14] and the previous section, though with $p=5$ and without $p+4$ branes, the $6 \mathrm{~d}$ world-volume theory becomes a $\mathcal{N}=(1,0)$ supersymmetric gauge theory with gauge group

$$
\prod_{\mu=0}^{r} U\left(K n_{\mu}\right)
$$

and matter multiplets in representations $\frac{1}{2} \oplus_{\mu \nu} a_{\mu \nu}\left(\square_{\mu}, \overline{\square_{\nu}}\right)$. In addition, there are $r$ $\mathcal{N}=(1,0)$ hypermultiplets and $r \mathcal{N}=(1,0)$ tensor multiplets (which combine into $r$ $\mathcal{N}=(2,0)$ matter multiplets), coming from reducing the $10 \mathrm{~d}$ two-form and four-form potentials down to $6 \mathrm{~d}$ on the $r$ cycles which generate $H_{2}$ of $\mathbb{C}^{2} / \Gamma_{G}$.

The above world-volume theory must be free of $6 \mathrm{~d}$ gauge anomalies. Writing the gauge group more generally as $\prod_{\mu=0}^{r} U\left(v_{\mu}\right)$, the 6 d gauge anomaly is

$$
\mathcal{A}=\widetilde{C}_{\mu \nu} v_{\nu} \operatorname{tr} F_{\mu}^{4}+3 \widetilde{C}_{\mu \nu} \operatorname{tr} F_{\mu}^{2} \operatorname{tr} F_{\nu}^{2}
$$

For $v_{\mu}=K n_{\mu}$ the deadly $\operatorname{tr} F_{\mu}^{4}$ irreducible anomaly terms vanish since $\widetilde{C}_{\mu \nu} n_{\nu}=0$. The remaining reducible anomaly in (4.2) can be cancelled with $P$ tensor multiplets provided it 
can be written as $\mathcal{A}=\sum_{i=1}^{P}\left(A_{i \mu} \operatorname{tr} F_{\mu}^{2}\right)^{2}$ with $A_{i \mu}$ real. The cancellation occurs by coupling the scalar fields $\Phi_{i}$ of the tensor multiplets to the gauge fields via the interactions

$$
\sum_{i=1}^{P} A_{i \mu} \Phi_{i} \operatorname{tr} F_{\mu}^{2} \quad \rightarrow \quad g_{\mu, e f f}^{-2}(\Phi)=g_{\mu, c l}^{-2}+\sum_{i} A_{i \mu} \Phi_{i}
$$

Thus, to cancel the remaining anomaly in (4.2), we want to write $\mathcal{A}=3 \widetilde{C}_{\mu \nu} x_{\mu} x_{\nu}$, where we define $x_{\mu} \equiv \operatorname{tr} F_{\mu}^{2}$, as a sum of squares. This is done for $A_{r}$ as

$$
\frac{1}{3} \mathcal{A}=\widetilde{C}_{\mu \nu} x_{\mu} x_{\nu}=\sum_{\mu=0}^{r}\left(x_{\mu}-x_{\mu+1}\right)^{2}, \quad x_{r+1} \equiv x_{0} .
$$

For $D_{r}$ this is done using

$$
\begin{aligned}
\frac{1}{6} \mathcal{A}=\frac{1}{2} \widetilde{C}_{\mu \nu} x_{\mu} x_{\nu} & =\left(x_{0}-\frac{1}{2} x_{2}\right)^{2}+\left(x_{1}-\frac{1}{2} x_{2}\right)^{2}+\frac{1}{2} \sum_{i=2}^{r-3}\left(x_{i}-x_{i+1}\right)^{2} \\
& +\left(\frac{1}{2} x_{r-2}-x_{r-1}\right)^{2}+\left(\frac{1}{2} x_{r-2}-x_{r}\right)^{2}
\end{aligned}
$$

For $E_{6}$, this is done using

$$
\begin{aligned}
\frac{1}{6} \mathcal{A}=\frac{1}{2} \widetilde{C}_{\mu \nu} x_{\mu} x_{\nu} & =\left(x_{1}-\frac{1}{2} x_{2}\right)^{2}+\left(x_{0}-\frac{1}{2} x_{6}\right)^{2}+\left(x_{5}-\frac{1}{2} x_{4}\right)^{2}+\frac{3}{4}\left(x_{2}-\frac{2}{3} x_{3}\right)^{2} \\
& +\frac{3}{4}\left(x_{6}-\frac{2}{3} x_{3}\right)^{2}+\frac{3}{4}\left(x_{4}-\frac{2}{3} x_{3}\right)^{2} .
\end{aligned}
$$

For $E_{7}$, we have

$$
\begin{aligned}
\frac{1}{6} \mathcal{A}=\frac{1}{2} \widetilde{C}_{\mu \nu} x_{\mu} x_{\nu} & =\left(x_{0}-\frac{1}{2} x_{1}\right)^{2}+\frac{3}{4}\left(x_{1}-\frac{2}{3} x_{2}\right)^{2}+\frac{2}{3}\left(x_{2}-\frac{3}{4} x_{3}\right)^{2}+ \\
& +\left(x_{6}-\frac{1}{2} x_{5}\right)^{2}+\frac{3}{4}\left(x_{5}-\frac{2}{3} x_{4}\right)^{2}+\frac{2}{3}\left(x_{4}-\frac{3}{4} x_{3}\right)^{2}+\left(x_{7}-\frac{1}{2} x_{3}\right)^{2} .
\end{aligned}
$$

For $E_{8}$, we have

$$
\begin{aligned}
\frac{1}{6} \mathcal{A}=\frac{1}{2} \widetilde{C}_{\mu \nu} x_{\mu} x_{\nu} & =\left(x_{1}-\frac{1}{2} x_{2}\right)^{2}+\frac{3}{4}\left(x_{2}-\frac{2}{3} x_{3}\right)^{2}+\left(x_{8}-\frac{1}{2} x_{3}\right)^{2}+\left(x_{0}-\frac{1}{2} x_{7}\right)^{2} \\
& +\frac{3}{5}\left(x_{4}-\frac{5}{6} x_{3}\right)^{2}+\frac{5}{8}\left(x_{5}-\frac{4}{5} x_{4}\right)^{2}+\frac{2}{3}\left(x_{6}-\frac{3}{4} x_{5}\right)^{2}+\frac{3}{4}\left(x_{7}-\frac{2}{3} x_{6}\right)^{2} .
\end{aligned}
$$

Throughout we label2 the nodes of the extended Dynkin diagrams as in table 16 of [23]. In each of the above cases, $\mathcal{A}$ is a sum of $r$ squares. Thus the reducible anomaly can

2 With this labeling, the Dynkin indices are: For $A_{r}$, all $n_{\mu}=1$. For $D_{r}, n_{\mu}=1$ for $\mu=$ $0,1, r-1, r$ and $n_{\mu}=2$ for $2 \leq \mu \leq r-2$. For $E_{6}, n_{\mu}=1$ for $\mu=0,1,5 ; n_{\mu}=2$ for $\mu=2,4,6$ and $n_{\mu}=3$ for $\mu=3$. For $E_{7}, n_{\mu}=1$ for $\mu=0,6 ; n_{\mu}=2$ for $\mu=1,5,7 ; n_{\mu}=3$ for $\mu=2,4$ and $n_{\mu}=4$ for $\mu=3$. For $E_{8}, n_{\mu}=1$ for $\mu=0 ; n_{\mu}=2$ for $\mu=1,7 ; n_{\mu}=3$ for $\mu=6,8 ; n_{\mu}=4$ for $\mu=2,5 ; n_{\mu}=5$ for $\mu=4$ and $n_{\mu}=6$ for $\mu=3$. 
be cancelled in every case with $r$ tensor multiplets. This is perfect because, as discussed above, the theory indeed has $r$ tensor multiplets. These $r$ tensors must couple to the gauge fields in (4.1) according to (4.3) with (4.4)- (4.8).

The diagonal $U(1)$ factor in $\prod_{\mu=0}^{r} U\left(K n_{\mu}\right)$ has no charged matter and decouples. The other $r U(1)$ factors do have charged matter and are thus anomalous. As in the discussion in [14,28], $r$ hypermultiplets are needed to cancel these anomalies. This again is perfect because, as discussed above, we have $r$ hypermultiplets, corresponding to the blowing up modes of the $\mathbb{C}^{2} / \Gamma_{G}$ singularity. They pair up with the $U(1)$ gauge fields to give them a mass and their expectation values effectively become Fayet-Iliopoulos parameters in the gauge group. As mentioned in sect. 2.1, the blowing up modes are also realized as FayetIliopoulos parameters in the analogous $\prod U\left(n_{i}\right) / U(1)$ hyper-Kahler quotient construction of 13] of the ALE geometry. This direct correspondence makes it obvious that our gauge theory (4.1) will be properly behave under smoothing the ALE space: the Fayet-Iliopoulos terms will Higgs (4.1) to exactly the right analog for the smoothed ALE space. The cancellation of the $U(1)$ anomalies using the $r$ hyper-multiplets and the cancellation of the reducible $\operatorname{tr} F_{\mu}^{2} \operatorname{tr} F_{\nu}^{2}$ anomalies using the $r$ tensor multiplets can be regarded as being related by a remnant of the larger supersymmetry of the full type IIB theory.

The theories (4.1) have a Higgs branch associated with giving expectation values to the matter fields in the $\frac{1}{2} \oplus_{\mu \nu} a_{\mu \nu}\left(\square_{\mu}, \square_{\nu}\right)$. On this branch, the gauge group is broken according to $K \rightarrow K-R$, for any $R=1 \ldots K$, along with an extra, decoupled, unbroken $U(R)_{D}$ subgroup. The $U(R)_{D}$ theory has a hypermultiplet in the adjoint representation and is diagonally embedded in all of the groups in (4.1). The index of the embedding of $U(R)_{D}$ in each $U\left(K n_{\mu}\right)$ gauge group is $n_{\mu}$ and thus $U(R)_{D}$ has a gauge coupling

$$
g_{D}^{-2}=\sum_{\mu} n_{\mu} g_{\mu}^{-2}
$$

The gauge couplings $g_{\mu}^{-2}$ depend on the tensor multiplet expectation values according to (4.3) with (4.4) - (4.8). In each case, though, it follows from $\widetilde{C}_{\mu \nu} n_{\nu}=0$ that the linear combination (4.9) is independent of the $\left\langle\Phi_{i}\right\rangle, i=1 \ldots r$, tensor multiplet expectation values.

The interpretation of the above part of the Higgs branch is that it corresponds to moving $R$ of the IIB five-branes away from the $\mathbb{C}^{2} / \Gamma_{G}$ singularity. The $U(R)_{D}$ theory with a hypermultiplet in the adjoint and constant gauge coupling is exactly the expected $\mathcal{N}=(1,1)$ theory for the five-branes away from the ALE singularity. Also, the $D$ terms 
for the single hypermultiplet mode labeling motion along this flat direction can always be mapped to exactly those of the theory of [13], described in sect. 2.1, which gives the $\mathbb{C}^{2} / \Gamma_{G}$ ALE space. In other words, the $R$ five-branes, which should be able to wander around any point on $\mathbb{C}^{2} / \Gamma_{G}$, indeed can.

The theory (4.1) for type IIB branes at the $\mathbb{C}^{2} / \Gamma_{G}$ singularity can be given an explicit orbifold construction, following [14]. The $r \mathcal{N}=(1,0)$ hyper and tensor multiplets come from the $r$ twisted conjugacy classes of $\Gamma_{G}$. Following the discussion in [12], the twisted tadpoles reproduce the spacetime anomaly (4.2), which led to $v_{\mu}=K n_{\mu}$.

As the simplest example, consider $K$ IIB five-branes at a $\mathbb{C}^{2} / \mathbb{Z}_{2} A_{1}$ ALE singularity. The world-volume theory is $U(K) \times U(K)$ with hypermultiplets in the $(\square, \bar{\square}) \oplus(\bar{\square}, \square)$. These theories were introduced in [18] as a class of theories which satisfy the anomaly cancellation condition when coupled to gravity. Here we see that the gauge anomaly can be cancelled even with gravity decoupled by including an extra tensor multiplet.

When the $\mathbb{C}^{2} / \mathbb{Z}_{2}$ singularity is not blown up and thus the $U(K) \times U(K)$ theory has no Fayet-Iliopoulos term, there is a direction in the Higgs branch where the matter fields $Q_{1}$ in the $(\square, \bar{\square})$, and $Q_{2}$ in the $(\bar{\square}, \square)$ have expectation values $\left\langle Q_{f}\right\rangle=\operatorname{diag}\left(\left\langle E_{f}\right\rangle \times 1_{R}, 0 \times 1_{K-R}\right)$ where $I_{R}$ is the $R \times R$ identity matrix, for any $1 \leq R \leq K$, and $E_{f}, f=1,2$, are hypermultiplets. This is a $D$-flat direction provided the $\left\langle E_{f}\right\rangle$ satisfy the $D$ term equations for $U(1)$ with two electrons, giving precisely the construction of [13], reviewed in sect. 2.1 , for the singular $\mathbb{C}^{2} / \mathbb{Z}_{2}$ ALE space. The Higgs branch associated with the above $\left\langle Q_{f}\right\rangle$ is thus the mode for moving $R$ five-branes away from the singularity. When the $\mathbb{C}^{2} / \mathbb{Z}_{2}$ singularity is resolved and thus the $U(K) \times U(K)$ theory has Fayet-Iliopoulos term, the above Higgs branch only occurs for $R=K$, with the $\left\langle E_{f}\right\rangle$ satisfying the $D$ term equations for $U(1)$ with two electrons and non-zero Fayet-Iliopoulos term. Since $\left\langle E_{f}\right\rangle \neq 0$, we find that $U(K) \times U(K)$ is necessarily Higgsed to $U(K)_{D}$ at a scale set by the blowing up parameter. Thus, as should be expected, we necessarily obtain the the standard $\mathcal{N}=(1,1)$ supersymmetric $U(K)$ on the five-branes when the singularity is resolved. There is a similar analysis for the theories (4.1) for general $\mathbb{C}^{2} / \Gamma_{G}$ singularities.

Following [7,8], there can be a non-trivial $6 \mathrm{~d}$ RG fixed point at the origin of the Coulomb branch provided all $g_{\mu, e f f}^{-2}(\Phi)$ are non-negative along some entire "Coulomb wedge" of allowed $\Phi_{i}, i=1 \ldots r$. This should be true even in the limit when all $g_{\mu, c l}^{-2} \rightarrow 0$ in order to obtain a RG fixed point theory at the origin. However since (4.9) is a constant independent of the $\left\langle\Phi_{i}\right\rangle$ on the Coulomb branch, at least one of the $g_{\mu, \text { eff }}^{-2}$ must become negative for large $\left\langle\Phi_{i}\right\rangle$. This corresponds to the fact that the $U(R)_{D}$ subgroup is always 
IR free. As in [8], we can always take the $U(K)$ corresponding to the extended Dynkin node $\mu=0$ to be the IR free theory, which means that this gauge group is un-gauged in the IR limit. It is then possible to choose a Coulomb wedge so that the remaining gauge

groups in (4.1) all have $g_{\mu, e f f}^{-2}(\Phi) \geq 0$ along the entire wedge even in the $g_{\mu, c l}^{-2} \rightarrow 0$ limit.

Thus for any $\mathbb{C}^{2} / \Gamma_{G}$, and for every $K$, these theories give $6 \mathrm{~d}$ non-trivial RG fixed points with $r$ tensor multiplets and gauge group $\prod_{\mu=1}^{r} S U\left(K n_{\mu}\right)$, where the $U(1) \mathrm{s}$ in (4.1) have been eliminated, as discussed above, by the anomaly cancellation mechanism, and the $\mu=0$ node gives a global rather than local $U(K)$ symmetry.

The simplest example, based on $K$ IIB five-branes at a $\mathbb{C}^{2} / \mathbb{Z}_{2}$ singularity, thus gives a $6 \mathrm{~d} \mathcal{N}=(1,0)$ supersymmetric theory with gauge group $S U(K), 2 K$ matter fields in the , and a tensor multiplet. There is a non-trivial RG fixed point, for all $K$, at the point where the tensor multiplet has zero expectation value. Another string theory realization of these theories was given in [10].

\section{5. $S O(N)$ instantons on ALE spaces}

As in sect. 2.2, we can have Wilson line group elements $\rho_{\infty} \in S O(N)$ representing $\Gamma_{G}$. Following the discussion in [28], the type I or heterotic gauge group is really $\operatorname{Spin}(32) / \mathbb{Z}_{2}$, where the $\mathbb{Z}_{2}$ is generated by the element $w$ in the center of $\operatorname{Spin}(32)$ which acts as -1 on the vector, -1 on the spinor of negative chirality, and +1 on the spinor of positive chirality. Because only representations with $w=1$ are in the $\operatorname{Spin}(32) / \mathbb{Z}_{2}$ string theory, the identity element $e \in \Gamma_{G}$ can be mapped to either the element 1 or $w$ in $\operatorname{Spin}(32)$. Here we will only consider the case $e \rightarrow 1$, which is the case with possible vector structure.

The basic difference from the $U(N)$ case is that $S O(N)$ is a real group. Starting from $U(N), S O(N)$ can be regarded as being obtained by modding out by the complex conjugation operation $*$; for example the $\mathbf{N}$ and $\overline{\mathbf{N}}$ representations of $U(N)$ are both mapped to the $\mathbf{N}$ representation of $S O(N)$. Properly understanding this operation of $*$, it will be fairly straightforward to modify the results reviewed in sect. 3 to the $S O(N)$ case. In the orientifold discussion of sect. 6 , modding out by the operation $*$ is, of course, the orientifold process.

It will be useful in what follows to group the set of all $\{\mu=0 \ldots r\}$ into the subsets as $\{\mu\}=\mathcal{R} \oplus \mathcal{P} \oplus \mathcal{C} \oplus \overline{\mathcal{C}} ; \Gamma_{G}$ representations $R_{\mu}$ with $\mu \in \mathcal{R}$ are real; representations $R_{\mu}$ with $\mu \in \mathcal{P}$ are pseudo-real; representations $R_{\mu}$ with $\mu \in \mathcal{C}$ are complex, with the complex conjugate representation $\overline{R_{\mu}}=R_{\bar{\mu}}$, with $\bar{\mu} \in \bar{C}$. In terms of the $S U(2)$ which 
contains $\Gamma_{G}$, the real representations have integer spin and the central element $C$ of $S U(2)$ is represented by $C=1$, while the pseudo-real representations have half-integer spin and the central element is represented as $C=-1$. Clearly, one dimensional representations are either real or complex, not pseudo-real. Using the connection between $\Gamma_{G}$ and the $A D E$ extended Dynkin diagrams, since $\chi_{Q} \times R_{\mu}=a_{\mu \nu} R_{\nu}$ and $\chi_{Q}$ has spin $j=\frac{1}{2}$, links of the diagram connect nodes with opposite values of $C$. The decompositions of the representations are as follows (with sets not listed empty). For $A_{r}$ with $r$ odd, $\mathcal{R}=$ $\left\{0, \frac{1}{2}(r+1)\right\}, \mathcal{C}=\left\{1, \ldots, \frac{1}{2}(r-1)\right\}, \bar{C}=\left\{\frac{1}{2}(r+3), \ldots, r\right\}$. For $A_{r}$ with $r$ even, $\mathcal{R}=\{0\}$, $\mathcal{C}=\left\{1, \ldots, \frac{1}{2} r\right\}, \bar{C}=\left\{\frac{1}{2} r+1, \ldots, r\right\}$. For $D_{r}$, with $r$ odd, $\mathcal{R}=\{0,1,2 s+1 \mid s=$ $\left.1, \ldots, \frac{1}{2}(r-3)\right\}, \mathcal{P}=\left\{2 s \mid s=1, \ldots, \frac{1}{2}(r-3)\right\}, \mathcal{C}=\{r-1\}, \bar{C}=\{r\}$. For $D_{r}$ with $r$ even, $\mathcal{R}=\left\{0,1, r-1, r, 2 s+1 \mid s=1 \ldots \frac{1}{2}(r-4)\right\}, \mathcal{P}=\left\{2 s \mid s=1, \ldots, \frac{1}{2}(r-2)\right\}$. For $E_{6}, \mathcal{R}=\{0,3\}, \mathcal{P}=\{6\}, \mathcal{C}=\{1,2\}, \bar{C}=\{4,5\}$. For $E_{7}, \mathcal{R}=\{0,2,4,6,7\}$, $\mathcal{P}=\{1,3,5\}$. For $E_{8}, \mathcal{R}=\{0,2,4,6,8\}, \mathcal{P}=\{1,3,5,7\}$.

A useful quantity here is the "Frobenius-Schur indicator," which is defined (see, e.g. [29]) to be +1 for real representations; -1 for representations which are not real but whose characters are real, i.e. pseudo-real representations; and 0 for representations with complex character. We thus define $S_{\mu} \equiv+1$ for $\mu \in \mathcal{R}, S_{\mu} \equiv-1$ for $\mu \in \mathcal{P}$, and $S_{\mu} \equiv 0$ for $\mu \in \mathcal{C}$ or $\overline{\mathcal{C}}$. By a theorem discussed e.g. in [29],

$$
S_{\mu}=\frac{1}{\left|\Gamma_{G}\right|} \sum_{g \in \Gamma_{G}} \chi_{\mu}\left(g^{2}\right) .
$$

The representation $\rho_{\infty}$ of $\Gamma_{G}$ in $S O(N)$ can be written as $\rho_{\infty}=\oplus_{\mu} w_{\mu} R_{\mu}$, where $n_{\mu} w_{\mu}=N$. In order for $\rho_{\infty}$ to be real, for every copy of a complex representation $R_{\mu}$ with $\mu \in \mathcal{C}$, there has to be a copy of the complex conjugate representation $\overline{R_{\mu}}=R_{\bar{\mu}}$, with $\bar{\mu} \in \overline{\mathcal{C}}$ : i.e $w_{\mu}=w_{\bar{\mu}}$. The pseudo-real representations also must satisfy a condition to fit in $S O(N)$ : because they are representations of $S U(2)$ rather than $S O(3)$, they need to be embedded in an $S O(4) \cong S U(2) \times S U(2)$ subgroup of $S O(N)$. This means that $w_{\mu}$ must be even for $\mu \in \mathcal{P}$. Finally, the real representations are already representations of $S O(3)$ and thus fit into $S O(N)$ without the need for any such doubling. The non-trivial $\rho_{\infty}$ breaks $S O(N)$ to a subgroup as

$$
S O(N) \rightarrow \prod_{\mu \in \mathcal{R}} S O\left(w_{\mu}\right) \times \prod_{\mu \in \mathcal{P}} S p\left(\frac{1}{2} w_{\mu}\right) \times \prod_{\mu \in \mathcal{C}} U\left(w_{\mu}\right)
$$


The relevant "small instanton" theory is a supersymmetric gauge theory (with eight super-charges) with gauge group

$$
\prod_{\mu \in \mathcal{R}} S p\left(v_{\mu}\right) \times \prod_{\mu \in \mathcal{P}} S O\left(v_{\mu}\right) \times \prod_{\mu \in \mathcal{C}} U\left(v_{\mu}\right)
$$

The group of (5.2) arises as a global symmetry of the gauge theory (5.3); thus the $S p\left(v_{\mu}\right)$ has $w_{\mu}$ half-hypermultiplets in the $\square_{\mu}$, the $S O\left(v_{\mu}\right)$ has $\frac{1}{2} w_{\mu}$ hypermultiplets in the $\square_{\mu}$, and the $U\left(v_{\mu}\right)$ has $w_{\mu}$ hypermultiplets in the $\square_{\mu}$. In addition, there are hypermultiplets corresponding to the links of the extended Dynkin diagram $\mathrm{B}$ : there are hypermultiplets in the $\frac{1}{4} \oplus_{\mu \nu} a_{\mu \nu}\left(\square_{\mu}, \square_{\nu}\right)$, where the sum runs over all $\mu=0 \ldots r$, with $\square_{\mu} \equiv \square_{\mu}$ for $\bar{\mu} \in \overline{\mathcal{C}}$. In the $\frac{1}{4} \oplus_{\mu \nu}$, one factor of $\frac{1}{2}$ is for a double counting in $\oplus_{\mu \nu}$ and the other is because either: $\mu \in \mathcal{R}$ and $\nu \in \mathcal{P}$ (or vice-versa) and thus the gauge group is $\operatorname{Sp}\left(v_{\mu}\right) \times S O\left(v_{\nu}\right)$ and the matter is in a half-hypermultiplet of $\left(\square_{\mu}, \square_{\nu}\right)$; or $\mu$ (or $\left.\nu\right) \in \mathcal{C}$, in which case the extra factor of $\frac{1}{2}$ is for a second double counting associated with $\bar{\mu}$ (or $\left.\bar{\nu}\right) \in \bar{C}$.

The $v_{\mu}$ data in (5.3) will be related to the physical data $w_{\mu}$ which specify $\rho_{\infty}$ by a relation of the form

$$
\widetilde{C}_{\mu \nu} V_{\nu}=w_{\mu}-D_{\mu}
$$

for some $D_{\mu}$ with $n \cdot D=n \cdot w=N$, and $V_{\mu} \equiv \operatorname{dim}\left(\square_{\mu}\right)$ (i.e. $V_{\mu}=2 v_{\mu}$ for $S p\left(v_{\mu}\right)$ and $V_{\mu}=v_{\mu}$ for $S O\left(v_{\mu}\right)$ and $\left.U\left(v_{\mu}\right)\right)$. Repeated indices run over all $\mu=0 \ldots r$, with $w_{\bar{\mu}} \equiv w_{\mu}$ and $V_{\bar{\mu}} \equiv V_{\mu}$ for $\bar{\mu} \in \overline{\mathcal{C}}$ and $\mu \in \mathcal{C}$. Using (2.5), the solution of (5.4) with $v_{0}=K$ is

$$
V_{\mu}=2 K n_{\mu}+G_{\mu \nu}\left(w_{\nu}-D_{\mu}\right)
$$

In analogy with results for $U(N)$ quiver gauge groups, we expect that the gauge theory (5.3) has a Higgs branch on which the gauge group can be completely broken by the Higgs mechanism provided $N \geq 3$ and all $D_{\mu} \geq 0$. In this case, the dimension of the Higgs branch is given by

$$
\operatorname{dim}\left(\mathcal{M}_{H}\right)=\frac{1}{4} V_{\mu}\left(w_{\mu}+D_{\mu}\right)-\frac{1}{2} V_{\mu} S_{\mu}
$$

3 The case of $\Gamma_{G}=\mathbb{Z}_{2 P+1}$ discussed in [14, 8 is a special case in that the group $U\left(v_{P}\right)$ also has a hypermultiplet in the $\bigoplus_{P}$. In terms of the discussion here, the unique aspect of $\Gamma_{G}=\mathbb{Z}_{2 P+1}$ is that the extended $S U(2 P+1)$ Dynkin diagram is the only case which does not admit a two coloring of the nodes, with linked nodes of opposite colors, corresponding to whether the corresponding representation of $S U(2)$ has integer or half-integer spin. The results below thus apply for every case except for $\Gamma_{G}=\mathbb{Z}_{2 P+1}$, which is covered by the analysis of [8]. 
where $S_{\mu}$ is the Frobenius-Schur indicator discussed above the theorem (5.1).

First, we argue that the Higgs branch $\mathcal{M}_{H}$ of the above gauge theory is isomorphic to the moduli space $\mathcal{M}_{\text {Inst }}$ of $S O(N)$ instantons on the ALE space, giving a hyper-Kahler quotient construction of this moduli space, when (5.4) is satisfied and

$$
D_{\mu}=N \delta_{\mu 0} \quad \text { (hyper-Kahler quotient) }
$$

A caveat is that $r-|\mathcal{C}|$ of the blowing up modes of the ALE space can not be turned on, they are frozen at zero. Next we argue that the small instanton theory (5.3) with the data (5.7) can not arise physically in the world-volume of five-branes at the ALE singularity as it would have a deadly anomaly. There is a simple modification of (5.7) for which the anomalies vanish:

$$
D_{\mu}=16 S_{\mu} \quad \text { (physical), }
$$

where we define $S_{\mu}$ as above. Note that the condition (5.8) in (5.4) properly gives $N=$ $w_{\mu} n_{\mu}=D_{\mu} n_{\mu}=16 S_{\mu} n_{\mu}=32$, where the identity $S_{\mu} n_{\mu}=2$ follows from (5.1) and character orthogonality, using $n_{\mu}=\chi_{\mu}(h=1)$ and $\sum_{g} \delta_{g^{2}, 1}=2$, since there are always precisely two elements, $g=1$ and the central element $C$ of $S U(2)$, which square to unity. As expected, consistency of the 5-brane theory requires 32 9-branes.

The instanton number (second Chern class) with a given $\rho_{\infty}$ is given by (2.3) with $\kappa=2$, corresponding to the index of the embedding of $S U(2)$ in $S O(N)$ (in an $S O(4) \cong$ $S U(2) \times S U(2))$. Using (5.5),

$$
I=\frac{1}{2\left|\Gamma_{G}\right|}\left(V \cdot n+G_{\mu \nu} D_{\mu} n_{\nu}\right)
$$

On the orientifold, $V \cdot n / 2\left|\Gamma_{G}\right|$ is the number of five-branes. In particular, with (5.7) the last term in (5.9) vanishes and the result (5.9) gives that the instanton number is the number of five-branes in the hyper-Kahler quotient case.

The dimension of the moduli space of $S O(N)$ instantons on the ALE space is given by (2.6) with $C_{2}(S O(N))=N-2$ and $\chi_{A d}\left(\rho_{\infty}\right)(g)=\frac{1}{2} w_{\mu} w_{\nu} \chi_{\mu}(g) \chi_{\nu}(g)-\frac{1}{2} w_{\mu} \chi_{\mu}\left(g^{2}\right)$, which follows because $\chi_{A d}\left(\rho_{\infty}\right)$ is the antisymmetric product of $\rho_{\infty} \times \rho_{\infty}$ for $S O(N)$. Thus

$$
\operatorname{dim}\left(\mathcal{M}_{\text {Inst }}\right)=(N-2) I+\frac{1}{2} w_{\mu} w_{\nu} X_{\mu \nu}-w_{\mu} F_{\mu}
$$

with $X_{\mu \nu}$ defined by (2.7) (and we used $w_{\bar{\nu}}=w_{\nu}$ ) and $F_{\mu}$ is defined by

$$
F_{\mu} \equiv \frac{1}{4\left|\Gamma_{G}\right|} \sum_{g \neq 1} \frac{\chi_{\mu}\left(g^{2}\right)-n_{\mu}}{2-\chi_{Q}(g)} \text {. }
$$


To evaluate $F_{\mu}$, note that

$$
\widetilde{C}_{\mu \nu} F_{\nu}=\frac{1}{4\left|\Gamma_{G}\right|} \sum_{g \neq 1}\left(\frac{2-\chi_{Q}\left(g^{2}\right)}{2-\chi_{Q}(g)}\right) \chi_{\mu}\left(g^{2}\right)=\frac{1}{2} S_{\mu}-\frac{n_{\mu}}{\left|\Gamma_{G}\right|}+\frac{1}{4\left|\Gamma_{G}\right|} \sum_{g} \chi_{Q}(g) \chi_{\mu}\left(g^{2}\right)
$$

where we used $2-\chi_{Q}\left(g^{2}\right)=\left(2-\chi_{Q}(g)\right)\left(2+\chi_{Q}(g)\right)$, which can be seen by $\chi_{Q}(g) \equiv 2 \cos \left(\theta_{g}\right)$, since then $\chi_{Q}\left(g^{2}\right)=2 \cos \left(2 \theta_{g}\right)$, and also (5.1). The last sum in (5.12) vanishes, as can be seen by noting that $\chi\left(g^{2}\right)=\chi_{S}(g)-\chi_{A}(g)$, where $S$ and $A$ are the symmetric and antisymmetric products; it follows from this that $\chi_{\mu}\left(g^{2}\right)$ has integer spin $j$ and thus has trivial projection on $\chi_{Q}(g)$, which has spin $j=\frac{1}{2}$. Therefore, using (2.5), we find $F_{\mu}=$ $\frac{1}{2} G_{\mu \nu} S_{\nu}-\left(\left|\Gamma_{G}\right|\right)^{-1} G_{\mu \nu} n_{\nu}$. Using this in (5.10), applying various other formulae appearing above, and comparing with (5.6), we find

$$
\operatorname{dim}\left(\mathcal{M}_{\text {Inst }}\right)=\operatorname{dim}\left(\mathcal{M}_{H}\right)-\frac{1}{2} G_{\mu \nu} S_{\mu} D_{\nu}+\frac{1}{4} G_{\mu \nu} D_{\mu} D_{\nu}
$$

In particular, with (5.7), $\operatorname{dim}\left(\mathcal{M}_{\text {Inst }}\right)=\operatorname{dim}\left(\mathcal{M}_{H}\right)$, giving a check of our conjecture that in this case $\mathcal{M}_{H} \cong \mathcal{M}_{\text {Inst }}$.

As a further check of $\mathcal{M}_{H} \cong \mathcal{M}_{\text {Inst }}$, it can be checked that the gauge theory (5.3) has a flat direction in the Higgs branch, associated with giving expectation values to the matter fields in the $\frac{1}{4} \oplus a_{\mu \nu}\left(\square_{\mu}, \square_{\nu}\right)$, which breaks the gauge group according to $V_{\mu} \rightarrow V_{\mu}-2 R n_{\mu}$, for any $R=1 \ldots K$, with an extra, decoupled, unbroken $S p(R)_{D}$ subgroup. The $S p(R)_{D}$ has the standard matter content of [1] , 16 $\square_{D} \oplus \square_{D}$, and is diagonally embedded in all of the groups in (5.3). The index of the embedding of $S p(R)_{D}$ in each group is $n_{\mu}$ and thus $S p(R)_{D}$ has gauge coupling

$$
g_{D}^{-2}=\sum_{\mu} n_{\mu} g_{\mu}^{-2}
$$

The interpretation of this part of the Higgs branch is that $R$ small instantons have been moved away from the $\Gamma_{G}$ orbifold singularity. Indeed, it appears that the $D$ terms for the single hypermultiplet Higgs mode labeling motion along this flat direction can always be mapped to exactly those of the theory of $\left[13\right.$, described in sect. 2.1 , which gives the $\mathbb{C}^{2} / \Gamma_{G}$ ALE space. In other words, the location of the center of the $R$ small $S O(32)$ instantons, which should be able to wander around on the entire $\mathbb{C}^{2} / \Gamma_{G}$ ALE space, indeed can.

Because of the $|\mathcal{C}| U\left(v_{\mu}\right)$ factors in (5.3), it is possible to turn on $|\mathcal{C}|$ Fayet-Iliopoulos terms, which gives the modification of the small instanton gauge group when the corresponding blowing up mode of the singularity is turned on. On the other hand, for the $S O\left(v_{\mu}\right)$ or $S p\left(v_{\mu}\right)$ factors in (5.3), there can be no such Fayet-Iliopoulos term and thus the 
corresponding blowing-up modes are frozen. The number of frozen blowing up modes is $r-|\mathcal{C}|$. For $D_{r}$ with $r$ even, $|\mathcal{C}|=0$ and thus all $r$ blowing up modes are frozen. For $D_{r}$ with $r$ odd, $|\mathcal{C}|=1$ and thus there are $r-1$ frozen blowing up modes. For $E_{6},|\mathcal{C}|=2$ and thus there are 4 frozen blowing up modes. For $E_{7}$ and $E_{8},|\mathcal{C}|=0$ and thus there are 7 and 8, respectively, frozen blowing up modes. These "missing" blowing up modes will be remembered shortly.

Despite the above evidence that $\mathcal{M}_{H} \cong \mathcal{M}_{\text {Inst }}$ when $D_{\mu}=N \delta_{\mu 0}$ (subject to the above caveat that some blowing up modes are frozen), these gauge theories can not arise in the world-volume of small $S O(N)$ instanton D5 branes sitting at the ALE singularity. Indeed, such theories have deadly $\operatorname{tr} F^{4}$ anomalies in the world-volume of the five-branes, which renders them inconsistent. This sickness can not be cured by adding more fields, even gravity. However, as in the $A_{r}$ case [8], there is a simple modification of the relation for which this deadly anomaly vanishes. The six-dimensional gauge anomaly for theories of the above type with general choices of $w_{\mu}$ and $v_{\mu}$ is given by

$$
\mathcal{A}=\frac{1}{2}\left(\widetilde{C}_{\mu \nu} V_{\nu}-w_{\mu}+16 S_{\mu}\right) \operatorname{tr} F_{\mu}^{4}+\frac{3}{2} \widetilde{C}_{\mu \nu} \operatorname{tr} F_{\mu}^{2} \operatorname{tr} F_{\nu}^{2}
$$

where $S_{\mu}$ is defined as above and $F_{\bar{\mu}} \equiv F_{\mu}$ along with $V_{\bar{\mu}} \equiv V_{\mu}$ and $w_{\bar{\mu}} \equiv w_{\mu}$ for $\bar{\mu} \in \overline{\mathcal{C}}$. Thus we conjecture that the physical gauge group living in the world-volume of the D5 branes is a gauge theory of the above type but with the relation (5.4) with (5.8), $D_{\mu}=16 S_{\mu}$.

Having cancelled the deadly $\operatorname{tr} F^{4}$ anomalies, the remaining anomaly in (5.15) can be cancelled by coupling the $6 \mathrm{~d}$ gauge theory to extra tensor multiplets, much as in the theories discussed in the previous section. Using (4.4) - 4.7), the reducible anomaly in (5.15) is the sum of $r$ square terms. However, with the identification $x_{\bar{\mu}} \equiv x_{\mu}$ for $\bar{\mu} \in \bar{C}$, $\mathcal{A}$ is actually the sum of $r-|\mathcal{C}|$ independent square terms. Thus the anomaly can be cancelled with $r-|\mathcal{C}|$ tensor multiplets. This leads to effective gauge couplings which vary over the Coulomb branch as in (4.3). As in the theories discussed in the previous section, it follows from $\widetilde{C}_{\mu \nu} n_{\nu}=0$ that the linear combination (5.14) is constant on the Coulomb branch. This is sensible as the $S p(R)_{D}$ of $R$ small instantons away from the singularity should have a constant gauge coupling.

Finally, the $|\mathcal{C}| U(1)$ factors are eliminated by the anomaly cancellation mechanism using the $|\mathcal{C}|$ hyper-multiplet blowing up parameters which remain massless on the Coulomb branch. 
With $D_{\mu}=16 S_{\mu}$, (5.9) gives for the number of five-branes on the orientifold

$$
\frac{1}{2\left|\Gamma_{G}\right|} V_{\mu} n_{\mu}=I-\frac{8}{\left|\Gamma_{G}\right|} G_{\mu \nu} n_{\mu} S_{\nu}=I-(r+1),
$$

Where the last identity for $G_{\mu \nu} n_{\mu} S_{\nu}$ can be verified in every case using the explicit expressions for the $G_{\mu \nu}$ in table 7 of [23]. The result (5.16) has a natural interpretation: $r+1$ is the Euler character of the ALE space $\mathbb{C}^{2} / \Gamma_{G_{r}}$, which is the instanton number associated with the standard embedding, when the gauge connection is set to equal the spin connection. Since five-branes carry $H$ charge, (5.16) is the standard relation giving the total (magnetic) $H$ chargel 3 as the second Chern class $I$ minus the Euler character. In particular, for the standard embedding there are no five-branes.

With $D_{\mu}=16 S_{\mu}$, it is no longer true that $\mathcal{M}_{H} \cong \mathcal{M}_{\text {Inst }}$. Indeed, even their dimensions differ: as seen from (5.13),

$$
\operatorname{dim}\left(\mathcal{M}_{H}\right)=\operatorname{dim}\left(\mathcal{M}_{\text {Inst }}\right)-56 G_{\mu \nu} S_{\mu} S_{\nu}=\operatorname{dim}\left(\mathcal{M}_{\text {Inst }}\right)-28(r-|\mathcal{C}|),
$$

where the last equality can be verified in every case using the explicit forms for the $G_{\mu \nu}$. We are thus missing $28(r-|\mathcal{C}|)$ hypermultiplet moduli associated with the gauge bundle. Combining these with the $r-|C|$ missing blowing-up modes, we see that a transition has occurred where $29(r-|\mathcal{C}|)$ hypermultiplets have been traded for $r-|\mathcal{C}|$ tensor multiplets. This is perfect, because $r-|\mathcal{C}|$ tensor multiplets is precisely what we needed to cancel the anomaly (5.15).

To summarize, we have presented evidence that small $S O(N)$ instantons on a $\mathbb{C}^{2} / \Gamma_{G}$ orbifold singularity have a "Coulomb branch" with $r-|\mathcal{C}|$ extra tensor multiplets and the theories (5.3) with (5.4), for $D_{\mu}=16 S_{\mu}$, arising in the world-volume of the D5 branes. Note that this Coulomb branch can only exist when all $V_{\mu}$, given by (5.5) with $D_{\mu}=16 S_{\mu}$, are non-negative, giving a lower bound on the allowed $K$.

As a simple concrete example, consider the $D_{4}$ case with trivial $\rho_{\infty}=1$. The $6 \mathrm{~d}$ brane world-volume gauge group (5.3) is $S p\left(v_{0}\right) \times S p\left(v_{1}\right) \times S O\left(v_{2}\right) \times S p\left(v_{3}\right) \times S p\left(v_{4}\right)$, with 32 half-hypermultiplets in the $\square_{0}$ and half-hypermultiplets in the $\left(\square_{\mu}, \square_{2}\right)$ for $\mu=0,1,3,4$.

4 Including gravity, the tensors also couple to the nine-brane gauge groups (5.2) according to the standard Green-Schwarz mechanism. When combined with the fact that $\int_{A L E} d H \neq 0$ with five-branes present, this means that there is an anomaly inflow mechanism, with net current flowing onto the brane from the ten-dimensional world. This is the limit of the mechanism of [30] where the instantons shrink to zero size. 
Using (5.5) and (5.8), $v_{0}=K, v_{1}=K-8, v_{2}=4 K-16, v_{3}=K-8, v_{4}=K-8$. In addition, there are the $r-|\mathcal{C}|=4$ extra tensor multiplets. Exactly this theory arose in the analysis of [11], via the $F$ theory description of compactification of the heterotic theory on a compact $K 3$, of the "collision of two hidden obstructors." It was conjectured in [11] that this theory should be related to a $D_{4}$ singularity. Here we see it as a special case of theories obtained for small $S O(32)$ instantons at arbitrary $\mathbb{C}^{2} / \Gamma_{G}$ singularities.

Following [7,8], there can be a non-trivial $6 \mathrm{~d}$ RG fixed point at the origin of the Coulomb branch provided all $g_{\mu, e f f}^{-2}(\Phi)$, given by (4.3) using (4.5) - (4.8), are non-negative along some entire "Coulomb wedge" of allowed $\Phi_{i}, i=1 \ldots r-|\mathcal{C}|$. This should be true even in the limit when all $g_{\mu, c l}^{-2} \rightarrow 0$ in order to obtain a RG fixed point theory at the origin. However, since (5.14) is a constant independent of the $\left\langle\Phi_{i}\right\rangle$ on the Coulomb branch, at least one of the $g_{\mu}^{-2}$ must become negative for large $\left\langle\Phi_{i}\right\rangle$. This corresponds to the fact that the $S p(R)_{D}$ subgroup is always IR free. As in [8], we can always take the $S p\left(v_{0}\right)$ corresponding to the extended Dynkin node to be the IR free theory, which means that this gauge group is un-gauged in the IR limit. It is then possible to choose a Coulomb wedge so that the remaining gauge groups in (5.3) all have $g_{\mu, e f f}^{-2}(\Phi) \geq 0$ along the entire wedge even in the $g_{\mu, c l}^{-2} \rightarrow 0$ limit. Thus each of our theories give an infinite family of $6 \mathrm{~d}$ non-trivial RG fixed points, labeled by $K$, with the $S p\left(v_{0}\right)$ factor in the gauge group (5.3) a global rather than gauge symmetry and the $U(1)$ factors eliminated by the anomaly cancellation mechanism.

\section{Orientifold construction of the theories}

Type I on the $\mathbb{C}^{2} / \Gamma_{G}$ singularity can be constructed via an orientifold of type IIB by including $\Omega$, the element that reverses the orientation, along with $\Gamma_{G}$ in the orbifold group; $\Omega^{2}=1$, and $\Omega g=g \Omega$. We must choose matrices $\gamma_{g}$ and $\gamma_{\Omega}$ to represent the action of $\Gamma_{G}$ and $\Omega$ on the Chan-Paton factors. The $\gamma_{g}$ must represent the $\Gamma_{G}$ group relations up to a phase. In addition, there are two more algebraic consistency conditions [31, 14]:

$$
\gamma_{\Omega}= \pm \gamma_{\Omega}^{T}
$$

where the upper(lower) sign is for $9(5)$ branes, and

$$
\gamma_{g} \gamma_{\Omega} \gamma_{g}^{T}=\gamma_{\Omega}
$$


Requiring the Chan Paton factor $\gamma_{C}$ corresponding to the central element $C=-1$ of $S U(2)$ to act as $\gamma_{C}=1$ in the trivial representation, there is no freedom for an extra phase in the gamma matrices and we should expect a single possibility, with 32 nine-branes.

The action of $\Gamma_{G}$ on the five-brane and nine-brane Chan-Paton factors is as in (3.6). The action of $\Omega$ on the five-branes is represented as

$$
\gamma_{\Omega, 5}=\oplus_{\mu \in \mathcal{R}}\left(I_{n_{\mu}} \otimes J_{V_{\mu}}\right) \oplus_{\mu \in \mathcal{P}}\left(J_{n_{\mu}} \otimes I_{V_{\mu}}\right) \oplus_{\mu \in C}\left(J_{2} \otimes I_{V_{\mu}}\right),
$$

where $J_{n}$, with $n$ even, is the $n \times n$ symplectic matrix: $J_{n}=-J_{n}^{T}$, with $J_{n}^{2}=-I_{n}$. In the last term in (6.3), $J_{2}$ acts to take $\mu \in \mathcal{C}$ to $\bar{\mu} \in \overline{\mathcal{C}}$. Similarly, $\Omega$ acts on nine-branes as

$$
\gamma_{\Omega, 9}=\oplus_{\mu \in \mathcal{R}}\left(I_{n_{\mu}} \otimes I_{w_{\mu}}\right) \oplus_{\mu \in \mathcal{P}}\left(J_{n_{\mu}} \otimes J_{w_{\mu}}\right) \oplus_{\mu \in \mathcal{C}}\left(\sigma_{1} \otimes I_{w_{\mu}}\right),
$$

where in the last term $\sigma_{1}$ is a symmetric Pauli matrix which exchanges $\mu \in \mathcal{C}$ with $\bar{\mu} \in \overline{\mathcal{C}}$. These yield precisely the gauge groups (5.3) for five-branes and (5.2) for nine-branes.

As always, the untwisted nine-brane tadpoles can be cancelled by having 32 ninebranes, i.e. $w \cdot n=32$. The tadpoles in the sector twisted by $g \neq 1$ can be written in the form:

$$
\frac{1}{2-\chi_{Q}(g)}\left(w_{\mu} \chi_{\mu}(g)-\left(2-\chi_{Q}(g)\right) v_{\mu} \chi_{\mu}(g)-16 \sum_{h} \delta_{g, h^{2}}\right)^{2} .
$$

Using the technique discussed in [12], we can extract the irreducible anomaly coefficient of $\operatorname{tr} F_{\mu}^{4}$ :

$$
-\left|\Gamma_{G}\right|\left(-\widetilde{C}_{\mu \nu} v_{\nu}+w_{\mu}-D_{\mu}\right)-n_{\mu}(32-w \cdot n),
$$

where

$$
D_{\mu} \equiv \frac{16}{\left|\Gamma_{G}\right|} \sum_{g} \sum_{h} \chi_{\mu}(g) \delta_{g, h^{2}}=16 S_{\mu}
$$

and we used (5.1). The $32-w \cdot n$ term in (6.6) vanishes by virtue of the untwisted nine-brane tadpole. We thus find perfect agreement with the irreducible space-time anomaly (5.15) of the five-brane gauge theory. The coefficient of $\operatorname{tr} F_{\mu}^{2} \operatorname{tr} F_{\nu}^{2}$ in the reducible anomaly is similarly obtained from (6.5), using the technique of [12], to be proportional to $\widetilde{C}_{\mu \alpha} \sum_{g \neq 1} \chi_{\alpha}(g) \chi_{\nu}(g)=\left|\Gamma_{G}\right| \widetilde{C}_{\mu \bar{\nu}}$, which agrees with the reducible anomaly in (5.15) (since $F_{\bar{\nu}}=F_{\nu}$ ).

To calculate the closed string spectrum, we note that the closed string partition function and Klein bottle splits into terms that are the Abelian groups representing the conjugacy classes. For all $\Gamma_{G}$ we can apply the result of [12] for the case with vector structure: 
we get a tensor from each twisted conjugacy class which is self-conjugate (i.e. contains its inverse) whereas, if it is conjugate to another twisted sector conjugacy class, we get a tensor and a hypermultiplet from the two twisted sectors. The number of conjugacy classes is the same as the number of irreps, which is $r+1$ for the $\Gamma_{G_{r}}$. One of these is the identity, so there are $r$ twisted sector conjugacy classes. Conjugacy classes containing their inverse have real character and thus there are $r-2|\mathcal{C}|$ self-conjugate classes and $|\mathcal{C}|$ remaining conjugate pairs. Thus we get $r-|\mathcal{C}|$ tensor multiplets and $|\mathcal{C}|$ hypermultiplet blowing up modes from the closed string twisted sectors. In agreement with the discussion in the previous section, we are missing $r-|\mathcal{C}|$ hypermultiplet blowing up modes (Fayet-Iliopoulos parameters) and instead have $r-|\mathcal{C}|$ tensor multiplets.

Finally, as discussed in $[12]$, the $r-|\mathcal{C}|$ tensor multiplets will have the correct couplings (4.3) to the gauge fields to cancel the reducible $\widetilde{C}_{\mu \nu} \operatorname{tr} F_{\mu}^{2} \operatorname{tr} F_{\nu}^{2}$ anomaly. Similarly, the $|\mathcal{C}|$ hypermultiplets have the correct couplings to cancel the $U(1)$ anomalies in the five-brane gauge group (5.3).

The above, perturbative, orientifold analysis applies far out along the "Coulomb branch," where the tensors have large expectation value and the theory is weakly coupled. As discussed in [12], the orientifold construction gives an intuitive picture for how the extra tensors of the Coulomb branch arise: starting from IIB on the ALE space and orientifolding to obtain type I, the expectation values of the tensor multiplets have an interpretation as distances of five-branes in the extra direction of $M$ theory. Arguments similar to [32], suggest that $M$ theory on $\mathbb{R}^{1} / \mathbb{Z}_{2}$ gives, at the origin, the infinite coupling limit of the type I $S O(32)$ ten-dimensional string theory. By "compactifying" this theory on the infinite volume ALE space, we can obtain a finitely coupled type I theory. In analogy with the $E_{8}$ case, we expect that a small $S O(32)$ instanton turns into an NS five-brane which can wander away from the origin into $\mathbb{R} / \mathbb{Z}_{2}$, with distance equal to the expectation value of the tensor multiplet.

Sufficiently far along the Coulomb branch, where the tensor multiplet has large expectation value, the theory is weakly coupled and we can apply type I perturbation theory. However, we can not blow up the ALE singularity (or turn on a $B$ field) because the corresponding hypermultiplet of type IIB was projected out. At the origin of the Coulomb branch there is the transition point to the Higgs branch, where the orbifold singularity can be blown up, but we can not continue to this branch via our perturbative type I orientifold analysis because the interactions at the origin are too strong. Via $S$ duality between the type I and heterotic $S O(32)$ strings in ten dimensions, there is a heterotic description with 
coupling $\lambda_{\text {heterotic }}=\frac{\mathcal{V}_{4}}{\lambda_{\text {typeI }}}$. For compact $\mathcal{V}_{4}$, it is thus possible to describe the Higgs branch, where $\lambda_{\text {typeI }}$ is large, in heterotic perturbation theory. In the non-compact case, $\lambda_{\text {heterotic }}$ blows up. The conclusion is that $M$ theory and type I perturbation theory are adequate to describe the Coulomb branch, while the Higgs branch is described by the perturbative heterotic string or $F$ theory.

\section{Other theories, with five-branes decoupled from the nine-branes}

There are other $6 \mathrm{~d}$ gauge theories of a type similar to those described in sect. 5 , but with no coupling to the nine-branes: i.e. all $w_{\mu}=0$. The theories are again based on moose diagrams which correspond to extended Dynkin diagrams and can be constructed for all $A_{r=2 M-1}, D_{r}, E_{6}$, and $E_{7}$ Dynkin diagrams. The basic difference from the theories discussed in sect. 5 can be regarded as a different action $*^{\prime}$ of the complex-conjugation operation. Much as in (5.3), the gauge group is

$$
\prod_{\mu \in \mathcal{R}^{\prime}} S p\left(v_{\mu}\right) \times \prod_{\mu \in \mathcal{P}^{\prime}} S O\left(v_{\mu}\right) \times \prod_{\mu \in \mathcal{C}^{\prime}} U\left(v_{\mu}\right)
$$

where we decompose the set of nodes of the extended Dynkin diagram into subsets as $\{\mu\}=\mathcal{R}^{\prime} \oplus \mathcal{P}^{\prime} \oplus \mathcal{C}^{\prime} \oplus \overline{\mathcal{C}^{\prime}}$, with the subsets in the various cases defined as follows (with sets not listed empty): For $A_{r=2 M-1}, \mathcal{R}^{\prime}=\{2 s \mid s=0 \ldots M-1\}, \mathcal{P}^{\prime}=\{2 s+1 \mid s=0 \ldots M-1\}$. For $D_{r}$ with $r$ odd, $\mathcal{R}^{\prime}=\left\{0,1,2 s+1 \mid s=1 \ldots \frac{1}{2}(r-3)\right\}, \mathcal{P}^{\prime}=\left\{r-1, r, 2 s \mid s=1 \ldots \frac{1}{2}(r-3)\right\}$. For $D_{r}$ with $r$ even, $\mathcal{R}^{\prime}=\left\{0,1,2 s+1 \mid s=1 \ldots \frac{1}{2}(r-4)\right\}, \mathcal{P}^{\prime}=\left\{2 s \mid s=1 \ldots \frac{1}{2}(r-2)\right\}, \mathcal{C}^{\prime}=$ $\{r-1\}, \overline{\mathcal{C}^{\prime}}=\{r\}$. For $E_{6}, \mathcal{R}^{\prime}=\{0,1,3,5\}, \mathcal{P}^{\prime}=\{2,4,6\}$. For $E_{7}, \mathcal{R}^{\prime}=\{0,6,7\}, \mathcal{P}^{\prime}=\{3\}$, $\mathcal{C}^{\prime}=\{1,2\}, \overline{\mathcal{C}^{\prime}}=\{4,5\}$. The gauge theory (7.1) has hypermultiplets corresponding to the links in the extended Dynkin diagrams: there are hypermultiplets in the $\frac{1}{4} \oplus_{\mu \nu} a_{\mu \nu}\left(\square_{\mu}, \square_{\nu}\right)$, where the factor in $\frac{1}{4} \oplus_{\mu \nu}$ is the same as explained after (5.3).

The six-dimensional gauge anomaly of the (7.1) is

$$
\mathcal{A}=\frac{1}{2}\left(\widetilde{C}_{\mu \nu} V_{\nu}+16 S_{\mu}^{\prime}\right) \operatorname{tr} F_{\mu}^{4}+\frac{3}{2} \widetilde{C}_{\mu \nu} \operatorname{tr} F_{\mu}^{2} \operatorname{tr} F_{\nu}^{2}
$$

where we define $S_{\mu}^{\prime} \equiv 1$ for $\mu \in \mathcal{R}^{\prime}, S_{\mu}^{\prime} \equiv-1$ for $\mu \in \mathcal{P}^{\prime}$, and $S_{\mu}^{\prime} \equiv 0$ for $\mu \in \mathcal{C}^{\prime}$ or $\overline{\mathcal{C}}^{\prime}$ and $F_{\bar{\mu}} \equiv F_{\mu}$ along with $V_{\bar{\mu}} \equiv V_{\mu} \equiv \operatorname{dim}\left(\square_{\mu}\right)$ and $w_{\bar{\mu}} \equiv w_{\mu}$ for $\bar{\mu} \in \overline{\mathcal{C}}$. The irreducible anomaly in (7.2) vanishes provided

$$
V_{\mu}=2 K n_{\mu}-16 G_{\mu \nu} S_{\nu}^{\prime},
$$


where $K$ is an arbitrary integer. This is similar to the expressions in sect. 5 except that here there are no hypermultiplets associated with coupling to nine-branes, all $w_{\mu}=0$. Indeed, introducing $w_{\mu}$ extra (half) hypermultiplets in the $\square_{\mu}$ would lead to a modification of (7.2) analogous to (5.15), but the requirement of canceling the irreducible $\operatorname{tr} F_{\mu}^{4}$ anomalies would then give $w_{\mu} n_{\mu}=0$, i.e. all $w_{\mu}=0$, because here $16 n_{\mu} S_{\mu}^{\prime}=0$ in every case rather than $16 n_{\mu} S_{\mu}=32$ as found in sect. 5. Finally, much as in sect. 5, the reducible anomaly in (7.2) can be cancelled by introducing $r-\left|\mathcal{C}^{\prime}\right|$ tensor multiplets.

The simplest example of these theories is the case associated with the $S U(2)$ extended Dynkin diagram. The gauge group (7.1) is $S p(K) \times S O(2 K+8)$ with two halfhypermultiplets in the $(\square, \square)$, corresponding to the two links in the diagram. $K$ is an arbitrary integer. These theories were presented in [18 as a class of theories which satisfy the anomaly cancellation conditions when coupled to gravity. Here we see that the anomaly can be cancelled even with gravity decoupled, by including an extra tensor multiplet. Another simple example is the case associated with the $D_{4}$ extended Dynkin diagram. The gauge group $(7.1)$ for $D_{4}$ is $S p(K)_{0} \times S p(K)_{1} \times S O(4 K+16) \times U(2 K+8)$ with half-hypermultiplets in the $\left(\mathbf{2} \mathbf{K}_{i}, \mathbf{4 K}+\mathbf{1 6}\right), i=0,1$, and a hypermultiplet in the $(\mathbf{4 K}+\mathbf{1 6}, \mathbf{2 K}+\mathbf{8})$, corresponding to the links. In addition, there are $r-\left|\mathcal{C}^{\prime}\right|=3$ tensor multiplets.

Each of the theories (7.1) with $(7.3)$ and $r-\left|\mathcal{C}^{\prime}\right|$ tensors lead to an infinite family of 6d RG fixed points, labeled by $K$. Again, it is necessary to take the $S p\left(v_{0}\right)$ factor in (7.1) to be a global rather than gauge symmetry to avoid Landau poles on the Coulomb branch. Also, the $U(1)$ factors are again eliminated.

\subsection{Orientifold construction of the theories}

The $A_{1}$ case discussed in [18] and above, with gauge group $S p\left(\frac{1}{2} V_{0}\right) \times S O\left(V_{1}\right)$, can be constructed via an $\Omega \mathbb{Z}_{4}$ orientifold. We take $\alpha=e^{2 \pi i / 4}$ the generator of $\mathbb{Z}_{4}$ and represent the generator of $\Omega \mathbb{Z}_{4}$ by

$$
\gamma_{\Omega \alpha}=\left(\begin{array}{cc}
J_{V_{0}} & 0 \\
0 & -I_{V_{1}}
\end{array}\right),
$$

where, as before, $J$ is the symplectic matrix and $I$ is the identity matrix. This satisfies the conditions needed for the case with vector structure so we expect a tensor from the $\mathbb{Z}_{2}$ twisted sector. There are no nine-branes or D5 branes since we do not have elements $\Omega$ or $\Omega \alpha^{2}$. Because there are no D5 branes or nine-branes, there normally would be no mechanism for cancelling crosscap terms by branes. However, if we assume the existence 
of "twisted" five-branes on which twisted open strings can end [19], we can cancel the crosscap terms. The tadpoles are found á la [33] to be

$$
\frac{1}{4 \sin ^{2} \frac{\pi}{2}}\left(4 \sin ^{2} \frac{\pi}{2}\left(V_{0}-V_{1}\right)+32\right)^{2}
$$

so $\widetilde{C}_{\mu \nu} V_{\nu}=-D_{\mu} \equiv 16(-1)^{\mu}$ for $\mu=0,1$, i.e. $V_{1}=V_{0}+8$ as expected.

This construction would not work for $A_{2 M-1}$ singularities with $M>1$ because there would be disk terms which could not be cancelled by crosscaps. However, the orientifold theories $\Omega(-1)^{F_{L}} R_{3} \times \mathbb{Z}_{2 M}$ studied by [19, 34], corresponding to $\mathrm{F}$ theory on $\left(\mathbb{C}^{2} / \mathbb{Z}_{2 M} \times\right.$ $\left.T^{2}\right) / \mathbb{Z}_{2}$, can be T-dualized to orientifolds with five-branes and nine-branes. There is no coupling and hence, no matter from the intersection of the two kinds of branes. Such a construction can yield the $A_{2 M-1}$ case of $(7.3)$ with $K=0$, which gives $V_{\mu \in \mathcal{R}^{\prime}}=0$ and $V_{\mu \in \mathcal{P}^{\prime}}=8$, i.e. $S O(8)^{\otimes M}$, and the required $2 M-1$ extra tensors. Indeed, this spectrum for the $M=1,2,3$ cases can easily be read off the results of [19], adjusted to the non-compact case. The solutions of [19] were not proven to be the most general solutions, and it would be interesting to see if all of the above models without coupling to nine-branes could be obtained from this construction. It would, of course, be necessary to include twisted open strings to cancel the tadpoles. Because of the lack of coupling to nine-branes, the fivebranes of these theories cannot be interpreted as small instantons and any heterotic dual description would have to be highly unusual.

\section{Building other $6 \mathrm{~d}$ moose models}

We will make some general observations about how to build $6 \mathrm{~d}$ theories for which the irreducible anomaly can be cancelled. Consider a theory with a product gauge group, with some matter fields coupling the representations. We focus on two factors out of the possibly much larger theory, groups $G_{1} \times G_{2}$, with some matter in representations $\left(r_{1}, r_{2}\right)$ coupling them.

Consider first the case of $U\left(v_{1}\right) \times U\left(v_{2}\right)$ coupled by $n_{c}$ hypermultiplets in the $(\square, \square)$. There could also be additional matter multiplets in either gauge group. In order to cancel the irreducible $\operatorname{tr} F_{i}^{4}$ anomalies, it is necessary to have $2 v_{1}-n_{c} v_{2}-P_{1}=0$ and $2 v_{2}-$ $n_{c} v_{1}-P_{2}=0$, where $P_{1} \geq 0$ are contributions associated with matter charged only under $U\left(v_{i}\right)$. In order to have $v_{i} \geq 0$, it is necessary to have $n_{c}=1$. The $v_{i}$ then satisfy $2 v_{1} \geq$ $v_{2} \geq \frac{1}{2} v_{1}$. Also, $P_{1}<2 v_{1}$ and $P_{2}<2 v_{2}$; thus the $U\left(v_{i}\right)$ can generally have matter only 
in the fundamental and/or two index symmetric or antisymmetric tensor representations. Further, it will generally not be possible to have other types of mixed representations. For example, if there were a matter field in the $(\square, \square)$ rather than in the $(\square, \square)$, it would be necessary to have $2 v_{1}-\frac{1}{2} v_{2}\left(v_{2}-1\right)=P_{1} \geq 0$ and $2 v_{2}-v_{1}\left(v_{2}-8\right)=P_{2} \geq 0$, which does not have a solution for $v_{2}>8$.

Consider next the case of $S O\left(v_{1}\right) \times S O\left(v_{2}\right)$ coupled with $n_{c}$ multiplets in the $(\square, \square)$ and possibly additional matter multiplets in either group. In order to cancel the irreducible $\operatorname{tr} F_{i}^{4}$ anomalies, it is necessary to have $v_{1}-8-n_{c} v_{2}-P_{1}=0$ and $v_{2}-8-n_{c} v_{1}-P_{2}=0$. For any $n_{c} \neq 0$, there is no solution of these equations with $v_{i} \geq 0$ if the $P_{i} \geq 0$, suggesting that $S O$ groups can never be coupled directly to other $S O$ groups. However there is a loophole here: spinors of $S O$ groups can lead to a negative contribution for the $P_{i}$ [35].

Consider next $S p\left(v_{1}\right) \times S p\left(v_{2}\right)$ coupled with $n_{c}$ multiplets in the ( $\left.\square, \square\right)$ and possibly additional matter in either group. In order to cancel the irreducible anomalies, it is necessary to have $2 v_{1}+8-2 n_{c} v_{2}=P_{1} \geq 0$ and $2 v_{2}+8-2 n_{c} v_{2}=P_{2} \geq 0$. For $n_{c}=1$, this requires $P_{1}+P_{2}=16$; this case was discussed in $[8]$ - it arises for small instantons at a $\mathbb{Z}_{2}$ orbifold singularity with possible vector structure. There can also be solutions with $n_{c}>1$ provided $P_{i}<8$.

For $U\left(v_{1}\right) \times S O\left(v_{2}\right)$ coupled with $n_{c}$ matter fields in the $(\square, \square)$ and possibly additional matter, the irreducible anomalies cancel provided $2 v_{1}-n_{c} v_{2}=P_{1} \geq 0$ and $v_{2}-8-n_{c} v_{1}=$ $P_{2} \geq 0$. It is thus necessary to have $n_{c} \leq 1$. For $U\left(v_{1}\right) \times S p\left(v_{2}\right)$ coupled with $n_{c}$ matter fields in the $(\square, \square)$ and possibly additional matter, the irreducible anomalies cancel provided $2 v_{1}-2 n_{c} v_{2}=P_{1} \geq 0$ and $v_{2}+8-n_{c} v_{1}=P_{2} \geq 0$, giving $n_{c} \leq 1$ and $P_{2} \geq$ 8. For $S O\left(v_{1}\right) \times S p\left(v_{2}\right)$ coupled with $n_{c}$ half-hypermultiplets in the $(\square, \square)$ and possibly additional matter, the irreducible anomalies cancel provided $v_{1}-8-n_{c} v_{2}=P_{1} \geq 0$ and $2 v_{2}+8-\frac{1}{2} n_{c} v_{1}=P_{2} \geq 0$, giving $n_{c} \leq 2$ and $P_{2} \geq 8$.

\section{Appendix A. Some $\Gamma_{G}$ representation theory}

We briefly discuss the representations of $\Gamma_{G}$ for $G=D_{r}$ and $G=E_{6}$ to illustrate the decompositions $\{\mu\}=\mathcal{R} \oplus \mathcal{P} \oplus \mathcal{C} \oplus \overline{\mathcal{C}}$ and the conjugacy classes (i.e. that there are $r-2|\mathcal{C}|$ classes which contain their own inverses and $|\mathcal{C}|$ pairs of classes which do not).

The $\Gamma_{G=D_{r}}$ dihedral group is generated by elements $\alpha$ and $\beta$ with $\alpha^{2}=\beta^{r-2}=C$ and $\beta \alpha \beta=\alpha$, where $C$ is the central element -1 of $S U(2)$. The four representations with 
$n_{\mu}=1$ are given by $\alpha_{\mu}=(-1)^{\mu}, \beta_{\mu}=1$ for $\mu=0,1$ and $\alpha_{\mu}=(-1)^{\mu} e^{i \pi r / 2}, \beta_{\mu}=-1$ for $\mu=r-1, r$. The representations with $n_{\mu}=2$ are given by

$$
\alpha_{\mu}=e^{i \pi(\mu-1) / 2}\left(\begin{array}{cc}
1 & 0 \\
0 & -1
\end{array}\right), \quad \beta_{\mu}=\left(\begin{array}{cc}
\cos \theta_{\mu} & \sin \theta_{\mu} \\
-\sin \theta_{\mu} & \cos \theta_{\mu}
\end{array}\right), \quad \theta_{\mu} \equiv \frac{\pi(\mu-1)}{r-2}
$$

$\mu=2, \ldots r-2$. For $D_{r}$ with $r$ even, the conjugacy classes are: [1], [-1], $\left[\beta^{k}, \beta^{-k}\right]$, $0 \leq k \leq r-2,[\alpha]$, and $[\alpha \beta]$, which are all self-conjugate (contain their inverse). For $r$ odd, the classes are the same but $[\alpha]$ and $[\alpha \beta]$ are now conjugate to each other (contain the other's inverses).

$\Gamma_{E_{6}}$ is generated by elements $\alpha, \beta$, and $\rho$ with $\alpha^{2}=\beta^{2}=\rho^{3}=C, \alpha \rho=\rho \beta \alpha, \beta \rho=\rho \alpha$, $\beta \alpha \rho=\rho \beta$, and $\beta \alpha \beta=\alpha$. The representations $\mu=0,1,5$ with $n_{\mu}=1$ have $\alpha_{\mu}=\beta_{\mu}=1$ and $\rho_{\mu}=1, \omega, \omega^{2}$, respectively, where $\omega \equiv e^{2 \pi i / 3}$. The representation $\mu=2$ has $\alpha_{\mu}=i \sigma_{1}$, $\beta_{\mu}=i \sigma_{3}$, and $\rho_{\mu}=\frac{e^{2 \pi i / 8}}{\sqrt{2}}\left(\begin{array}{cc}-i & -i \\ -1 & 1\end{array}\right)$ and is pseudo-real. The other two representations with $n_{\mu}=2, \mu=4$ and $\mu=6$, differ from $\mu=2$ in that $\rho_{4}=\omega \rho_{2}$ and $\rho_{6}=\omega^{2} \rho_{2}$; these two representations are complex conjugates of each other. Finally, the representation $\mu=3$ is given by

$$
\alpha_{3}=\left(\begin{array}{ccc}
-1 & 0 & 0 \\
0 & -1 & 0 \\
0 & 0 & 1
\end{array}\right) \quad \beta_{3}=\left(\begin{array}{ccc}
-1 & 0 & 0 \\
0 & 1 & 0 \\
0 & 0 & -1
\end{array}\right) \quad \rho_{3}=\left(\begin{array}{lll}
0 & 1 & 0 \\
0 & 0 & 1 \\
1 & 0 & 0
\end{array}\right)
$$

The conjugacy classes of $\Gamma_{E_{6}}$ are $[1],[-1],[\rho],\left[\rho^{2}\right],[\alpha],[-\rho]$, and $\left[-\rho^{2}\right]$, with $[\rho]$ and $\left[\rho^{2}\right]$ conjugate and $[-\rho]$ and $\left[-\rho^{2}\right]$ conjugate.

\section{Acknowledgments}

The work of J.B. is supported in part by NSF PHY-9513835. The work of K.I. is supported by NSF PHY-9513835, the W.M. Keck Foundation, an Alfred Sloan Foundation Fellowship, and the generosity of Martin and Helen Chooljian. 


\section{References}

[1] E. Witten, hep-th/9511030, Nucl. Phys. B 460 (1995) 541.

[2] M. Duff, R. Minasian, and E. Witten, hep-th/9601036, Nucl. Phys. B 465 (1996) 413.

[3] N. Seiberg and E. Witten, hep-th/9603003, Nucl. Phys. B 471 (1996) 121.

[4] M.J. Duff, H. Lu, and C.N. Pope, hep-th/9603037, Phys. Lett. B 378 (1996) 101.

[5] O. Ganor and A. Hanany, hep-th/9602120.

[6] E. Witten, hep-th/9609159, Mod. Phys. Lett. A11 (1996) 2649.

[7] N. Seiberg, hep-th/9609161.

[8] K. Intriligator, hep-th/9702038, Nucl Phys. B to appear

[9] U.H. Danielsson, G. Feretti, J. Kalkkinen, and P. Stjernberg, hep-th/9703098.

[10] M. Bershadsky and C. Vafa, hep-th/9703167.

[11] P. S. Aspinwall, hep-th/9612108.

[12] J. D. Blum, K. Intriligator, hep-th/9605030.

[13] P.B. Kronheimer, Jour. Differential Geometry, 29 (1989) 665.

[14] M. Douglas and G. Moore, hep-th/9603167.

[15] C. V. Johnson and R. C. Myers, hep-th/9610140.

[16] P. B. Kronheimer and H. Nakajima, Math. Ann. 288 (1990) 263.

[17] H. Georgi, Nucl. Phys. B 266 (1986) 274.

[18] J. H. Schwarz, hep-th/9512053, Phys. Lett. B 371 (1996) 223.

[19] J. D. Blum, hep-th/9608053, Nucl. Phys. B 486 (1997) 34.

[20] P. S. Aspinwall and D. R. Morrison, RU-97-29, IASSNS-HEP-97/46, to appear.

[21] M.R. Douglas, hep-th/9612126.

[22] M.F. Atiyah and I.M. Singer, Ann. Math. 87 (1968) 485, Ann. Math. 93 (1971) 119.

[23] R. Slansky, Physics Reports 79 (1981) 1.

[24] M.F. Atiyah, V.K. Patodi, and I.M. Singer, Math. Proc. Camb. Phil. Soc. 77 (1975) $43 ; 77$ (1975) 405; 79 (1976) 71.

[25] E. Witten, hep-th/9510135, Nucl. Phys. B 460 (1996) 335.

[26] M. Douglas, hep-th/9512077.

[27] P.S. Aspinwall, hep-th/9507012, Phys. Lett. B 357 (1995) 329.

[28] M. Berkooz, R.G. Leigh, J. Polchinski, J. Schwarz, N. Seiberg, and E. Witten, hepth/9605184, Nucl. Phys. B 475 (1996) 115.

[29] M.J. Collins, Representations characters of finite groups, Cambridge Studies in Advanced Mathematics 22, Cambridge University Press, 1990.

[30] J. Blum and J. Harvey, hep-th/9310035, Nucl. Phys. B 416 (1994) 119.

[31] E. G. Gimon and Polchinski, hep-th/9601038, Phys. Rev. D 54 (1996) 1667.

[32] P. Horava and E. Witten, hep-th/9510209, Nucl. Phys. B 460 (1996) 506.

[33] E. G. Gimon and C. V. Johnson, hep-th/9604129, Nucl. Phys. B 477 (1996) 715.

[34] J. D. Blum and A. Zaffaroni, Phys. Lett. B 387 (1996) 71.

[35] J. Erler, hep-th/9304104, J. Math. Phys. 35 (1994) 1819. 